jedenfalls am besten entsprechen, wenn man zur Reduction der Schur'schen Distanzen nur den constanten Messungsfehler $a=+0.23^{2}$ in Rechnung stellt.

Bei Ambronn wird die Darstellung der Bedingungsgleichungen sowohl durch $a$ allein als auch durch $a$ und $b$ zusammen verbessert, die Verbesserung ist aber nur sehr gering, namentlich in Anbetracht der Grösse von $\varepsilon$ selbst; auch haben die $\mathrm{m}$. F. von $a$ und $b$ diesen Grössen gegen. über sehr erhebliche Beträge. Das Vorhandensein von merklichen systematischen Fehlern in der Ambronn'schen Messungsreihe ist sonach ziemlich unwahrscheinlich.

Leipzig, 1898 September 16.

Bruno Peter.

\title{
Beobachtung der Venusbedeckung von 1898 Mai 22 auf der Sternwarte in Kiel.
}

Beobachter

F. Möller

F. Ristenpart

E. H. Schütz

P. Harzer
Instrument

Refractor

Cometensucher

A equatoreal

Passageninstrument

Oeffn.
$216^{\mathrm{mm}}$
133
117
67

Vergr.

Coordinaten gegen den Meridiankreis
Eintritt M. Z. Kiel

$\begin{array}{cc}\text { I. Eingriff bemerkt } & \text { Venus verschwindet } \\ 7^{\mathrm{h}} 20^{\mathrm{m}} 57^{\mathrm{s}} .4 & 7^{\mathrm{h}} 2 \mathrm{I}^{\mathrm{m}} 23^{5.8} \\ 60.1 & 22.4 \\ 59.0 & 22.9 \\ 57.8 & 21.2\end{array}$

Bei dem Austritte war der Mond durch Cirri unsichtbar und die Austrittsstelle der Venus konnte deshalb am Cometensucher und am Passageninstrument nicht festgehalten werden. Herr Möller sah durch die Cirri hindurch $8^{\mathrm{h}}{ }_{12}{ }^{\mathrm{m}}{ }_{5} \mathrm{I}^{\mathrm{s}}{ }^{7}$ die Venus halbirt, $8^{\mathrm{h}}{ }_{1} 3^{\mathrm{m}} 1^{\mathrm{s}}: 6$ die letzte Berührung. Herr Schütz sah $8^{\mathrm{h}}{ }_{1} 3^{\mathrm{m}} 15^{\mathrm{s}} \cdot 6$ die Venus mit ihrem nächsten Rande um die Hälfte ihres Durchmessers vom Mondrande entfernt.

Kiel, 1898 August.

P. Harzer.

Cometa $1898 \ldots$ (Brooks Ottobre 20)

osservata ad Arcetri coll' equatoriale di Amici. ${ }^{*}$ )

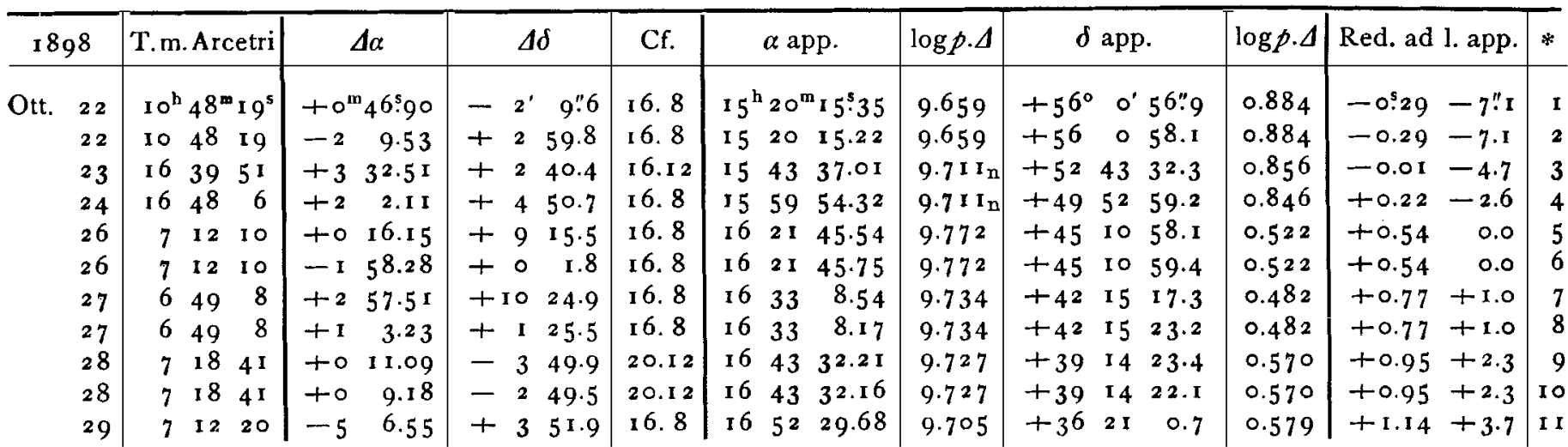

Ottobre 22, 23. Splendido. - Ottobre 24. Cielo torbido. - Ottobre 26, 27, 28, 29. Splendido ma chiaro di luna. La Cometa apparve in tutte le sere quale una macchia tonda del solito splendore nebuloso, del diametro di oltre un minuto primo d'arco, senza traccia di coda.

Stelle di confronto.

\begin{tabular}{|c|c|c|c|c|c|c|c|}
\hline$*$ & $\alpha$ I 898.0 & $\delta 1898.0$ & Autorità & $*$ & $\alpha$ I 898.0 & $\delta \simeq 898.0$ & Autorità \\
\hline$x$ & $15^{\mathrm{h}} \times 9^{\mathrm{m}} 28^{\mathrm{s}} \cdot 74$ & $+5^{6^{\circ}} 3^{\prime}$ 1 $3^{\prime \prime} 6$ & AG. Hels. 8337 & 7 & $16^{\mathrm{h}} 30^{\mathrm{m}}$ I $0^{\mathrm{s}} \cdot 26$ & $+42^{\circ} 4^{\prime} 51.4$ & AG. Bonn $1060 \mathrm{I}$ \\
\hline 2 & $15 \quad 22 \quad 25.04$ & $+555^{8} 5.4$ & AG. Hels. $83^{6} 3$ & 8 & 16324.17 & +42 I 356.7 & $\mathrm{BB} \mathrm{VI}+4292727$ \\
\hline 3 & I $540 \quad 4.5$ I & $+5^{2} 4^{\circ} 5^{6.6}$ & AG. Cambr. $4^{821}$ & 9 & I 64320.17 & +3918 11.0 & AG. Lund Z. 245,253 \\
\hline 4 & $\begin{array}{lll}15 & 57 & 51.99\end{array}$ & +4948 1 1.1 & AG. Bonn 10275 & 10 & 164322.03 & $+39 \times 79.3$ & AG. Lund Z. 245,253 \\
\hline 5 & $1621 \quad 28.85$ & +45 I 42.6 & AG. Bonn 10515 & I 1 & I6 $57 \quad 35.09$ & $+3^{6} 17$ & $A G$. Lund $Z .412,413$ \\
\hline 6 & $\begin{array}{lll}16 & 23 & 43.49\end{array}$ & +451057.6 & AG. Bonn $1053^{6}$ & & & & \\
\hline
\end{tabular}

Arcetri-Firenze, 1898 Novembre I.

A. Abetti.

*) Obbiettivo $284 \mathrm{~mm}$, Micrometro a lamine I 9."45 . Ingrandimento 124 (vedi Pubbl. di Arcetri Fas. 9 pag. 3 nota 5). 